\section{Caso clínico: Neoplasia de células dendríticas plasmocitoides blásticas con respuesta inicial a quimioterapia y recaída en sistema nervioso central}

\author{
LORETO CONTRERAS ${ }^{1,2}$, LORETO MERCADO ${ }^{3}$, CAROLINA DELGADO $^{1,2}$, \\ CLAUDIA CABEZAS ${ }^{1}$, LAKSMI STARKE ${ }^{1}$, MÓNICA ROMERO ${ }^{3}$, \\ FERNANDO IBIETA ${ }^{3}$, MAURICIO HENRÍQUEZ ${ }^{2,3}$, MAURICIO CHANDIA ${ }^{1,2,3}$
}

Blastic plasmacytoid dendritic cell neoplasm (BPDCN) is a rare, clinically aggressive hematologic malignancy that most commonly manifests as cutaneous lesions with or without bone marrow involvement and leukemic dissemination. The demonstration of tumor cells with the characteristic immunophenotype with expression of CD56, generally CD4 and dendritic cell antigens (CD123, cyTCL-1, HLA-DR), in the absence of myeloid or lymphoid lineage markers is required for the diagnosis. Responses to chemotherapy are initially satisfactory, with frequent systemic and central nervous system relapses. We report a 24 year-old male with BPDCN, initially diagnosed and treated as non-Hodgkin CD4+ T-cell lymphoma, with initial complete remission who evolved with early central nervous system relapse. A second attempt of chemotherapy failed and the patient died two months later.

(Rev Med Chile 2017; 145: 115-120)

Key words: Hematologic neoplasms; immunophenotyping; Dentritic Cells, Plasmocytoid.

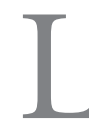

a neoplasia de células dendríticas plasmocitoides blásticas (NCDPB) es una neoplasia hematológica poco frecuente, clínicamente muy agresiva, que puede manifestarse como lesiones cutáneas con o sin compromiso de médula ósea o diseminación leucémica. Ha sido categorizada en la clasificación 2008 de la Organización Mundial de la Salud (OMS) dentro de las leucemias agudas y neoplasias derivadas de precursores hematopoyéticos mieloides ${ }^{1}$. La frecuencia de la enfermedad es muy baja $(<1 \%$ del total de neoplasias hematológicas), existiendo a la fecha sólo un caso publicado en nuestro país ${ }^{2}$. Por otro
'Unidad de Anatomía Patológica, Hospital "Dr. Guillermo Grant Benavente", Concepción, Chile. 2Facultad de Medicina, Universidad de Concepción, Concepción, Chile.

'Unidad de Hematología, Hospital "Dr. Guillermo Grant Benavente", Concepción, Chile.

Recibido el 8 de enero de 2016, aceptado el 7 de septiembre de 2016

Correspondencia a:

Dr. Mauricio Chandía Cabas Laboratorio de Citometría de Flujo. Hospital Guillermo Grant Benavente.

Víctor Lamas $s / n$ Edificio de Anatomía, Universidad de Concepción, Barrio Universitario, Concepción, Chile. mchcabas@gmail.com lado, las manifestaciones clínicas y fenotípicas se confunden con las de otras patologías oncohematológicas, por lo cual el diagnóstico definitivo es tardío. En la actualidad, existe evidencia creciente de que las NCDPB tienen su contrapartida normal en precursores de las células dendríticas plasmocitoides ( $\mathrm{p}-\mathrm{CD})$, que son presentadoras de antígeno profesionales originadas en la médula ósea $^{3}$. Clínicamente, se caracterizan por afección inicialmente sólo cutánea, con compromiso de médula ósea en la mayoría de los casos durante la evolución de la enfermedad. La respuesta al tratamiento es inicialmente satisfactoria, pero 
el desenlace clínico es desfavorable con recaídas refractarias a terapia y con frecuente compromiso del sistema nervioso central.

En el diagnóstico y sub-clasificación de la enfermedad es fundamental la contribución de técnicas complementarias a la morfología como la inmunohistoquímica y la citometría de flujo ${ }^{4}$. A continuación presentamos un caso clínico de NCDPB, inicialmente diagnosticado y tratado como linfoma no Hodgkin T CD4+, con remisión completa inicial, que posteriormente evoluciona con recaída precoz en sistema nervioso central.

\section{Caso clínico}

Paciente de 24 años, con antecedentes de amaurosis izquierda congénita, que consulta por cuadro de lesiones cutáneas solevantadas, eritema- tosas, violáceas y difusas en cuero cabelludo, dorso y tórax, asociado a anemia normocítica de causa no precisada. La biopsia de piel fue informada como linfoma no Hodgkin $T$ de células pequeñas y medianas, con patrón de infiltración nodular y difuso, y expresión de CD4 en 90\% de las células tumorales (Figura 1). Se realizó estudio de diseminación que demostró ausencia de adenopatías en tomografía axial computada de tórax, abdomen y pelvis e infiltración de médula ósea en biopsia (Figura 1). Se planificaron 8 ciclos de quimioterapia CHOP (ciclofosfamida, doxorrubicina, vincristina y prednisona), de los cuales sólo pudo realizar 6 por mala tolerancia, presentando en todos los ciclos vómitos y cefalea persistentes, que requirieron uso de múltiples antieméticos. Presentó resolución completa de lesiones cutáneas y de compromiso de médula ósea en la biopsia, por lo que se declaró en remisión completa. Por persistencia de cefalea y

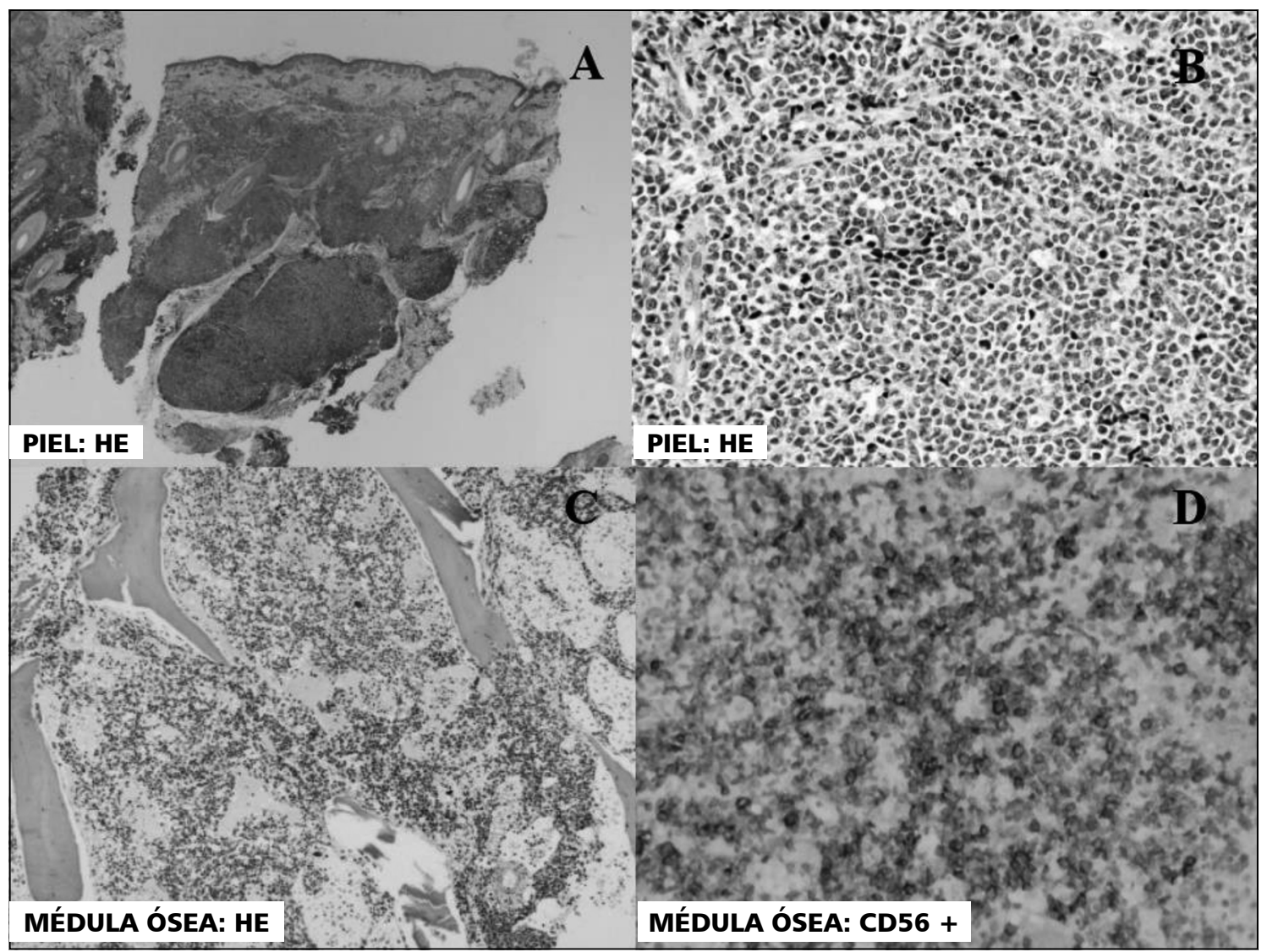

Figura 1. A y B. Piel: infiltrado de células monomorfas en cuero cabelludo (A: H\&E, 2,5; B: H\&, 20 X). C y D. Médula ósea: infiltrado de células monomorfas (C: H\&E, 2,5 X) y expresión de CD56 (D: H\&E, 20 X). 
Neoplasia de células dendríticas plasmocitoides - L. Contreras et al

vómitos se realizó resonancia magnética cerebral que no evidenció lesiones. El estudio del líquido cefalorraquídeo (LCR) mostró hiperproteinorraquia y aumento de celularidad (Tabla 1), mientras que el inmunofenotipo fue informado como sugerente de infiltración de sistema nervioso central por NCDPB (Figura 2). Se planificó terapia de rescate con metotrexato intratecal más citarabina

Tabla 1. Estudios analíticos realizados durante la evolución del paciente

\begin{tabular}{|c|c|c|c|}
\hline & Diagnóstico & Fin de tratamiento & Recaída \\
\hline Tiempo desde el diagnóstico & & 6 meses & 7 meses \\
\hline Hemoglobina (g/dL) & 9,8 & 12,4 & 14,4 \\
\hline Hematocrito (\%) & 28,4 & 36,4 & 40,1 \\
\hline Plaquetas (x10e3/uL) & 206 & 311 & 296 \\
\hline Leucocitos (x10e3/uL) & 2,5 & 4,47 & 5,45 \\
\hline Sodio (mEq/L) & 138 & & 136 \\
\hline Potasio (mEq/L) & NA & & 3,8 \\
\hline Cloro (mEq/L) & 106 & & 101 \\
\hline BUN (mg/dL) & 6 & & 17 \\
\hline Albúmina (g/dL) & 4,10 & & 5,40 \\
\hline Creatinina (mg/dL) & 0,67 & 0,77 & 1,03 \\
\hline Calcio (mg/dL) & 9,5 & & 10,6 \\
\hline Fósforo (mg/dL) & N/A & & \\
\hline Fosfatasa alcalina (U/L) & 164 & & 116 \\
\hline LDH (U/L) & $\mathrm{N} / \mathrm{A}$ & & 299 \\
\hline$P C R(m g / d L)$ & 1,3 & & 1,0 \\
\hline TP (seg) & 11,50 & & 12,30 \\
\hline INR & 1,00 & & 1,10 \\
\hline TTPA (seg) & 34,10 & & 26,10 \\
\hline LCR: ADA (U/L) & & & 8,8 \\
\hline Proteinorraquia (g/L) & & & 0,96 \\
\hline Glucorraquia (g/dL) & & & 0,45 \\
\hline Leucocitos $\left(x \mathrm{~mm}^{3}\right)$ & & & 1350 \\
\hline
\end{tabular}
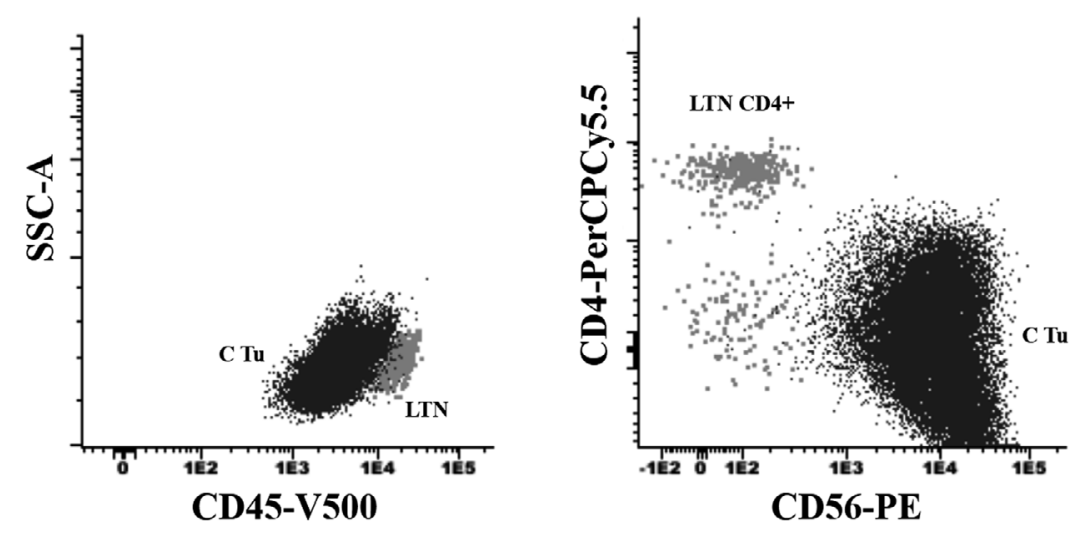

Figura 2. Inmunofenotipo por citometría de flujo de las células tumorales en líquido cefalorraquídeo en el momento de la recaída. A. Expresión débil a negativa de CD45. B. Coexpresión de CD4 (débil) y CD56. CTu: células tumorales, LTN: linfocitos T normales. 
en altas dosis, presentando como complicación 2 episodios de neutropenia febril de alto riesgo, sin aislamiento microbiológico, que fueron tratados con terapia antibiótica asociada y evolución favorable. Logró negativización inicial del LCR en el inmunofenotipo, pero posteriormente se comprobó persistencia de células patológicas.

A las 12 semanas de iniciada la quimioterapia de rescate, presentó dolor en glúteo izquierdo, irradiado a muslo y zona lumbar. Se realizó resonancia nuclear magnética de columna dorsolumbar que mostró infiltración por enfermedad de base en múltiples cuerpos vertebrales, huesos sacro e ilíaco izquierdos. Se consideró no candidato a radioterapia de sistema nervioso central dado lo difuso de la infiltración y se decidió manejo conservador, falleciendo 2 meses más tarde.

\section{Discusión}

La NCDPB fue descrita por primera vez en 1994 en un hombre de 63 años, con compromiso cutáneo, de médula ósea y sistema nervioso central al diagnóstico y expresión de CD4 y CD56 en las células neoplásicas; los autores la interpretaron como una variante del linfoma cutáneo ${ }^{5,6}$. Posteriormente aparecen reportes de nuevos casos con otras denominaciones como "leucemia aguda agranular de células NK", "linfoma de células NK blástico" y "tumores hematodérmicos CD4+/ CD56+", antes de la denominación actual de NCDPB de la clasificación de la OMS de $2008^{1}$.

Clínicamente, suele afectar más a hombres que a mujeres en una razón 2,5:1. La edad de presentación se concentra en adultos mayores, alrededor de los 65 años $^{7}$, pero puede presentarse también en adultos jóvenes, tal como ocurrió en este caso. La presentación más común es con lesiones cutáneas, las cuales pueden ser únicas o múltiples, con aspecto violáceo-marrón, pseudo-purpúricas, en placas o solevantadas que en un tercio de los casos consisten en 1-2 nódulos, mientras que en el resto de los casos puede afectar múltiples áreas. Las regiones más comprometidas por lesiones únicas son la cara, la región escapular y en menor medida el tronco y extremidades ${ }^{7}$. El compromiso de medula ósea, con o sin expresión leucémica, está presente en la mayoría de los pacientes en algún momento de la enfermedad y en casos muy poco frecuentes ha sido descrito en ausencia de lesio- nes cutáneas ${ }^{8}$. En etapas avanzadas puede existir compromiso de otros parénquimas como hígado, amígdalas, senos paranasales, órbitas y sistema nervioso central ${ }^{9}$. En nuestro caso, el paciente al ingreso presentaba lesiones en cuero cabelludo, dorso y tórax alto con características similares a las previamente descritas. El hemograma en más de dos tercios de los casos muestra trombocitopenia y anemia y en menor medida neutropenia'. En nuestro caso, estas características estaban presentes desde el ingreso, probablemente secundarias al compromiso demostrado de médula ósea.

La biopsia de piel muestra un infiltrado de células de tamaño mediano monomorfas que respeta la epidermis, pero puede extenderse al tejido celular subcutáneo, en ausencia de zonas de necrosis, presencia de células inflamatorias o invasión vascular. Las células son pobremente diferenciadas, con cromatina laxa y 2 o 3 nucléolos, con citoplasma agranular y levemente basófilo ${ }^{1}$. El inmunofenotipo de las células tumorales se puede estudiar por medio de la inmunohistoquímica o citometría de flujo, dependiendo del material disponible. Habitualmente se detecta co-expresión de CD4 y CD56 en ausencia de marcadores específicos de línea mieloide (mieloperoxidasa), linfoide $\mathrm{T}$ (CD3, CD5) y linfoide B (CD19, CD20 y CD79a). El linaje de $\mathrm{p}-\mathrm{CD}$ es demostrado por la expresión de marcadores como el CD123 (intenso), BCA2/ CD303 y cyTCL-1, junto a HLA-DR, el cual está presente por la calidad de célula presentadora de antígeno de la p-CD. Otros marcadores presentes en frecuencia variable son el CD36, CD68, CD7, desoxinucleotidil transferasa terminal (TdT) y CD33. Habitualmente no expresan marcadores de inmadurez como CD34 y CD117 y tampoco CD13 y CD16. Otro marcador específico de p-CD descrito en el último tiempo es el CD2AP ${ }^{1,10}$. En nuestro caso, el diagnóstico de la enfermedad fue retrospectivo y sugerido a partir de los hallazgos por inmunofenotipo del LCR efectuado durante la recaída precoz que presentó luego de la terapia inicial (Figura 2). Asimismo, la inmunohistoquímica de las muestras de médula ósea y piel realizadas al diagnóstico resultó también positivas para $\mathrm{CD} 4 \mathrm{y}$ CD56 (Figura 1). El diagnóstico diferencial de esta enfermedad debe hacerse con otras neoplasias que expresen CD56 y que puedan tener compromiso cutáneo o sistémico, como leucemias agudas mieloblásticas CD56+, linfomas T cutáneos CD4+, linfoma $\mathrm{T}$ tipo paniculitis, linfoma $\mathrm{T}$ cutáneo 
primario gamma-delta y linfoma NK/T tipo nasal ${ }^{1}$. Cerca de dos tercios de los pacientes con NCDPB presentan alteraciones cariotípicas, aunque no son de carácter diagnóstico ${ }^{11}$. Leroux et al. demostraron que la mayoría de los casos presenta deleciones en los cromosomas $5 \mathrm{q} 21$ o $5 \mathrm{q} 34$ (72\%), 12p13 (64\%), 13q13-q21 (64\%), 6q23-qter (50\%), 15q $(43 \%)$ y $9(28 \%)$ y alteraciones genéticas esporádicas que afectan los genes RB1, LATS2, CDKN1B, $C D K N 2 A$ y $T P 53^{12}$. Sapienza et al. estudiaron el perfil de expresión génica de 27 casos de NCDPB, encontrando una sobreexpresión de 2 targets del NF-kB targets (BCL2 e IRF4), que fue inhibido in vitro con bortezomib, lo que abre una oportunidad para la terapia con este agente ${ }^{13}$.

El tratamiento óptimo de las NCDPB no está establecido. La baja frecuencia de la enfermedad, la falta de una definición de consenso para el diagnóstico y la gran diversidad de tratamientos empleados hacen compleja la realización de ensayos aleatorizados. Independientemente del tratamiento empleado, el pronóstico de la enfermedad es ominoso y las sobrevidas reportadas son en su mayoría menores de 12 meses. La experiencia más grande publicada a la fecha es la de un grupo italiano en 43 pacientes con NCDPB y expresión leucémica, donde se demostraron mejores tasas de remisión completa y medianas de sobrevida global en pacientes tratados con esquemas de quimioterapia tipo leucemias linfoblástica/linfomas agresivos, en comparación con los tratados con esquemas de leucemias agudas mieloblásticas $(12,3 \text { vs } 7,1 \text { meses })^{14}$. Como terapia de consolidación se ha utilizado el trasplante alogénico en quienes son candidatos aceptables, reportándose medianas de sobrevida mayores que las de la quimioterapia. En el estudio italiano anteriormente mencionado se reportó una mediana de sobrevida global de 22,7 meses ${ }^{14}$. El trasplante autólogo en primera remisión completa se ha empleado con éxito en algunas series, reportándose sobrevidas globales y libres de progresión incluso similares al trasplante alogénico ${ }^{15}$, por lo que Pagano et al. lo han recomendado como consolidación para todos aquellos pacientes jóvenes no candidatos a trasplante alogénico ${ }^{16}$. Considerando la alta expresión de CD123 en las NCDPB, se ha desarrollado un conjugado de IL-3 asociada a toxina de difteria (SL-401) que se ha utilizado como terapia diana en trabajos fase $1 / 2^{17}$. Otra alternativa explorada es el uso de 5-azacitidina en 2 casos de NCDPB asociadas a mielodisplasia ${ }^{18}$. Basado en resultados in vitro se espera explorar a futuro opciones como lenalidomida ${ }^{19}$ y bortezomib ${ }^{13}$, como ya se comentó anteriormente.

Hemos reportado el caso de un paciente joven con un cuadro de lesiones cutáneas difusas eritemato-violáceas inicialmente catalogadas como linfoma $\mathrm{T}$, con compromiso de médula ósea al diagnóstico, que tras recibir terapia y presentar una recaída en sistema nervioso central, se hace el diagnóstico retrospectivo de una NCDPB, basados en la expresión de CD56 en la histología e inmunofenotipo. Sugerimos siempre tener en consideración la NCDPB en el diagnóstico diferencial de las patologías con compromiso cutáneo y expresión de CD56.

\section{Referencias}

1. Facchetti F, Jones DM, Petrella T. Blastic plasmacytoid dendritic cell neoplasm. En: Swerdlow SH, Campo E, Harris NL, eds. WHO Classification of Tumors of Haematopoietic and Lymphoid Tissues. Lyon: International Agency for Research on Cancer (IARC); 2008. p 145.

2. Tapia E O, Kam C, Rifo L, Villaseca H. Neoplasia blástica de células dendríticas plasmocitoides: Caso clínico. Rev Med Chile 2012; 140 (10): 1321-4.

3. Chaperot L, Bendriss N, Manches O, Gressin R, Maynadie M, Trimoreau F, et al. Identification of a leukemic counterpart of the plasmacytoid dendritic cells. Blood 2001; 97 (12): 3210-7.

4. Martin-Martin L, López A, Vidriales B, Caballero MD, Silva A, Ferreira S, et al. Classification and clinical behavior of blastic plasmacytoid dendritic cell neoplasms according to their maturation-associated immunophenotypic profile. Oncotarget 2015; 6 (22): 19204-16.

5. Adachi M, Maeda K, Takekawa M, Hinoda Y, Imai K, Sugiyama S, et al. High expression of CD56 (N-CAM) in a patient with cutaneous CD4-positive lymphoma. Am J Hematol 1994; 47: 278-82.

6. Petrella T, Bagot M, Willemze R, Beylot-Barry M, Vergier B, Delaunay M, et al. Blastic NK-cell lymphomas (agranular CD4+CD56+ hematodermic neoplasms): a review. Am J Clin Pathol 2005; 123 (5): 662-75.

7. Julia F, Petrella T, Beylot-Barry M, Bagot M, Lipsker $\mathrm{D}$, Machet $\mathrm{L}$, et al. Blastic plasmacytoid dendritic cell neoplasm: clinical features in 90 patients. Br J Dermatol 2013; 169 (3): 579-86.

8. Rauh MJ, Rahman F, Good D, Silverman J, Brennan 
MK, Dimov N, et al. Blastic plasmacytoid dendritic cell neoplasm with leukemic presentation, lacking cutaneous involvement: Case series and literature review. Leuk Res 2012; 36 (1): 81-6.

9. Feuillard J, Jacob MC, Valensi F, Maynadie R, Gressin $\mathrm{R}$, Chaperot L, et al. Clinical and biologic features of CD4(+), CD56(+) malignancies. Blood 2002; 99 (5): 1556-63.

10. Marafioti T, Paterson JC, Ballabio E, Reichard KK, Tedoldi S, Hollowood K, et al. Novel markers of normal and neoplastic human plasmacytoid dendritic cells. Blood 2008; 111 (7): 3778-92.

11. Riaz W, Zhang L, Horna P, Sokol L. Blastic plasmacytoid dendritic cell neoplasm: update on molecular biology, diagnosis, and therapy. Cancer Control 2014; 21 (4): 279-89.

12. Leroux D, Mugneret F, Callanan M, Radford-Weiss I, Dastugue N, Feuillard J, et al. CD4(+), CD56(+) DC2 acute leukemia is characterized by recurrent clonal chromosomal changes affecting 6 major targets: a study of 21 cases by the Groupe Francais de Cytogenetique Hematologique. Blood 2002; 99 (11): 4154-9.

13. Sapienza MR, Fuligni F, Agostinelli C, Tripodo C, Righi S, Laginestra MA, et al. Molecular profiling of blastic plasmacytoid dendritic cell neoplasm reveals a unique pattern and suggests selective sensitivity to NF-kB pathway inhibition. Leukemia 2014; 28 (8): 1606-16.
14. Pagano L, Valentini CG, Pulsoni A, Fisogni S, Carluccio $\mathrm{P}$, Mannelli F, et al. Blastic plasmacytoid dendritic cell neoplasm with leukemic presentation: an Italian multicenter study. Haematologica 2013; 98 (2): 239-46.

15. Aoki T, Suzuki R, Kuwatsuka Y, Kako S, Fujimoto K, Taguchi J, et al. Long-term survival following autologous and allogeneic stem cell transplantation for blastic plasmacytoid dendritic cell neoplasm. Blood 2015; 125 (23): 3559-62.

16. Pagano L, Valentini CG, Grammatico S, Pulsoni A. Blastic plasmacytoid dendritic cell neoplasm: diagnostic criteria and therapeutical approaches. Br J Haematol 2016; 174 (2): 188-202.

17. Frankel AE, Woo JH, Ahn C, Pemmaraju N, Medeiros BC, Carraway HE, et al. Activity of SL-401, a targeted therapy directed to interleukin-3 receptor, in blastic plasmacytoid dendritic cell neoplasm patients. Blood 2014; 124 (3): 385-92.

18. Laribi K, Denizon N, Ghnaya H, Atlassi M, Besançon A, Pineau-Vincent F, et al. Blastic plasmacytoid dendritic cell neoplasm: the first report of two cases treated by 5-azacytidine. Eur J Haematol 2014; 93 (1): 81-5.

19. Agliano A, Martin-Padura I, Marighetti P, Gregato G, Calleri A, Prior C, et al. Therapeutic effect of lenalidomide in a novel xenograft mouse model of human blastic NK cell lymphoma/blastic plasmacytoid dendritic cell neoplasm. Clin Cancer Res 2011; 17 (19): 6163-73. 